\title{
Representation of Head-Centric Flow in the Human Motion Complex
}

\author{
Jeroen Goossens, ${ }^{1,2}$ Sean P. Dukelow, ${ }^{3}$ Ravi S. Menon, ${ }^{4}$ Tutis Vilis, ${ }^{5}$ and Albert V. van den Berg 6 \\ ${ }^{1}$ Department of Biophysics, Radboud University Nijmegen Medical Centre, 6500 HB Nijmegen, The Netherlands, ${ }^{2}$ F. C. Donders Centre for Cognitive \\ Neuroimaging, 6500 HB Nijmegen, The Netherlands, ${ }^{3}$ Graduate Program in Neuroscience, Siebens-Drake Research Institute, University of Western \\ Ontario, London, Ontario, Canada N6G 2V4, ${ }^{4}$ Advanced Imaging Labs, The John P. Robarts Research Institute, London, Ontario, Canada N6A 5K8, \\ ${ }^{5}$ Department of Physiology and Pharmacology, University of Western Ontario, London, Ontario, Canada N6A 5C1, and ${ }^{6} \mathrm{Helmholtz}$ Institute, Functional \\ Neurobiology, University of Utrecht, 3584 CH Utrecht, The Netherlands
}

Recent neuroimaging studies have identified putative homologs of macaque middle temporal area (area MT) and medial superior temporal area (area MST) in humans. Little is known about the integration of visual and nonvisual signals in human motion areas compared with monkeys. Through extra-retinal signals, the brain can factor out the components of visual flow on the retina that are induced by eye-in-head and head-in-space rotations and achieve a representation of flow relative to the head (head-centric flow) or body (body-centric flow).

Here, we used functional magnetic resonance imaging to test whether extra-retinal eye-movement signals modulate responses to visual flow in the human MT + complex. We distinguished between MT and MST and tested whether subdivisions of these areas may transform the retinal flow into head-centric flow.

We report that interactions between eye-movement signals and visual flow are not evenly distributed across MT + . Pursuit hardly influenced the response of MT to flow, whereas the responses in MST to the same retinal stimuli were stronger during pursuit than during fixation. We also identified two subregions in which the flow-related responses were boosted significantly by pursuit, one overlapping part of MST. In addition, we found evidence of a metric relation between rotational flow relative to the head and fMRI signals in a subregion of MST. The latter findings provide an important advance over published single-cell recordings in monkey MST. A visual representation of the rotation of the head in the world derived from head-centric flow may supplement semicircular canals signals and is appropriate for cross-calibrating vestibular and visual signals.

Key words: heading; optic flow; pursuit eye movements; head movements; reference frames; spatial perception; vestibular

\section{Introduction}

Humans can accurately perceive their direction of self-motion from visual flow even during eye and head rotations (Gibson, 1966; Royden et al., 1992; Warren, 1995; Bardy and Warren, 1997; Beintema and van den Berg, 2001). This is remarkable because, for the rotating eye, the center of the radial flow on the retina no longer coincides with the heading direction (Gibson, 1966; Regan and Beverley, 1982; van den Berg and Beintema, 2000). Psychophysical studies indicate that humans cope with this rotation problem using visual and extra-retinal signals about the rotation of the eye (Warren and Hannon, 1988; Royden et al., 1994; van den Berg, 1996; Crowell et al., 1998; Lappe et al., 1999). Through the extra-retinal signal, the brain can compensate for this rotation and achieve a representation of flow relative to the

\footnotetext{
Received Sept. 13, 2005; revised April 11, 2006; accepted April 11, 2006.

This work was supported by Human Frontiers Science Program Grants SF0037/2000-B (J.G.) and RG0071/2000 (A.V.v.d.B., T.V., R.S.M.) and the Canadian Institutes of Health Research (S.P.D.).

Correspondence should be addressed to Jeroen Goossens, Department of Biophysics, Radboud University Nijmegen Medical Centre, G. Grooteplein 21, P.0. Box 9101, 6500 HB Nijmegen, The Netherlands. E-mail: j.goossens@science.ru.nl.

D0I:10.1523/JNEUROSCI.0730-06.2006

Copyright $\odot 2006$ Society for Neuroscience $\quad$ 0270-6474/06/265616-12\$15.00/0
}

head or body that allows for accurate heading judgments (Perrone, 1992; Beintema and van den Berg, 1998; Lappe, 1998; Zemel and Sejnowski, 1998; Hanada, 2005).

Electrophysiological studies have identified several motionselective areas in monkeys, some of which are involved in this compensation for eye rotation. Two well studied areas are the middle temporal (MT) and medial superior temporal (MST) regions in the superior temporal sulcus. Area MT has strong projections to adjacent area MST (Maunsell and van Essen, 1983; Desimone and Ungerleider, 1986). MT neurons have relatively small receptive fields and carry purely visual signals that encode the basic elements of motion. Neurons in MST, conversely, have large receptive fields that prefer certain patterns of visual motion (Duffy and Wurtz, 1991; Orban et al., 1992; Graziano et al., 1994; Lappe et al., 1996), and their responses are often modulated by eye position (Bremmer et al., 1997), eye velocity (Bradley et al., 1996; Page and Duffy, 1999), and vestibular signals (Shenoy et al., 1999). This integration of visual and nonvisual signals in MST could make it a crucial area for the perception of self-motion (Britten and van Wezel, 1998; Page and Duffy, 2003).

Also the human temporal cortex contains a region $(\mathrm{MT}+)$ with visual motion (de Jong et al., 1994; Tootell et al., 1995a,b; 
Rees et al., 2000; Huk and Heeger, 2002) and smooth-pursuit related signals (Petit and Haxby, 1999; Tanabe et al., 2002). Recent functional magnetic resonance imaging (fMRI) studies have shown involvement of MT + in heading estimation (Peuskens et al., 2001) and the presence of an area sensitive to optic flow patterns (Morrone et al., 2000). Moreover, two distinct subdivisions of $\mathrm{MT}+$, which differ in retinotopic organization and receptive field size, have been identified as homologs of macaque MT and MST (Dukelow et al., 2001; Huk et al., 2002). However, very little is known about the integration of visual and nonvisual signals in $\mathrm{MT}+$. Our fMRI study aims to fill this gap. We combined expanding flow patterns that simulate forward movement of the eye with sinusoidal rotation of the gaze line, which adds rotational flow to the expansion. These stimuli were presented during steady fixation and during sinusoidal pursuit. We adjusted the motion pattern on the screen so as to keep the retinal flow pattern the same. Briefly, we find that pursuit signals boost the blood oxygenation level-dependent (BOLD) responses to expanding retinal flow and that the BOLD signals of a subdivision of putative area MST increase as a function of the speed of the rotational flow relative to the head.

\section{Materials and Methods}

Subjects. Ten healthy human volunteers (six males and four females) with normal vision were paid for their participation. They all gave informed consent in writing, and the study was given ethical approval by University of Western Ontario Ethics committee. All subjects were well practiced in fixation and smooth pursuit eye movement tasks, and they had participated previously in other functional magnetic resonance imaging studies.

Data acquisition. Images were collected with a 4.0 tesla Varian (Palo Alto, CA)/Siemens (Erlangen, Germany) Unity Inova whole-body imaging system equipped with whole-body shielded gradients. BOLD functional data were collected using $\mathrm{T} 2{ }^{*}$-weighted echo-planar imaging. To provide enhanced signal-to-noise, a custom-built quadrature radio frequency surface coil ( $8 \mathrm{~cm}$ diameter) was placed unilaterally over the right occipital region, centered on $\mathrm{MT}+$.

At the beginning of each session, the location of MT + was determined using a low-resolution functional localizer scan [72 volumes; in-plane resolution of $3.0 \mathrm{~mm}$; $64 \times 64$; field of view $(\mathrm{FOV}), 19.2 \mathrm{~cm} ; 115-\mathrm{mm}$ slices oriented perpendicular to the calcarine sulcus; $1.95 \mathrm{~s}$ volumeacquisition time; time to repetition (TR), $0.49 \mathrm{~s}$; four shots; time to echo (TE), $15.0 \mathrm{~ms}$. During this run, the subjects viewed alternating stationary and moving dots (16 s epochs, $144 \mathrm{~s}$ total). The localizer data were then quickly analyzed using a motion minus stationary comparison [Stimulate software (Strupp, 1996)] to ensure that the higher-resolution slices would encompass area MT+.

In subsequent experimental runs, high-resolution slices were centered on $\mathrm{MT}+$, as prescribed by the localizer, and acquired with an in-plane resolution of $1.1 \mathrm{~mm}$ and slice thickness of $2 \mathrm{~mm}(128 \times 128$; FOV, 14 $\mathrm{cm}$; 11 slices; $4 \mathrm{~s}$ volume-acquisition time; TR, $1.0 \mathrm{~s}$; four shots; TE, 15.0 $\mathrm{ms})$. Fifty-six volumes were acquired in each of these functional scans. At the end of each session, high-resolution inversion-prepared threedimensional (3D) T1-weighted anatomical images (either 32 or 64 slices; $256 \times 256$; TR, $12.5 \mathrm{~ms}$; TE, $6.5 \mathrm{~ms}$ ) were collected.

In a separate session, subjects were rescanned using a birdcage-style head coil to obtain full-brain anatomical images. A high-resolution inversion prepared $3 \mathrm{D}$ T1-weighted sequence was used (voxel size, 0.86 $\mathrm{mm}$ in-plane; $256 \times 256$; FOV, $22 \mathrm{~cm}$; 256 slices; TR, 11.5-12 ms; TE, $5.5-6.0 \mathrm{~ms})$.

Visual stimulation. Visual stimuli were generated on an Apple Macintosh G4 PowerPC (Apple Computers, Cupertino, CA) using OpenGl rendering software. The stimuli were presented on a translucent screen (Da-Lite, Warsaw, IN) via a liquid crystal display projector (NEC MT800, $800 \times 600$ resolution) and a series of lenses. Subjects lay supine in the magnet and viewed the images back-projected through a mirror placed $\sim 5 \mathrm{~cm}$ in front of their eyes. The room and bore were completely dark with only visible structure on the screen. The stimuli were viewed at an effective distance of $15 \mathrm{~cm}$ and subtended a visual angle of $90^{\circ}$ wide $X$ $30^{\circ}$ tall.

In the test conditions, motion through a cloud (dimensions, $50 \mathrm{~m}$ wide $\times 17 \mathrm{~m}$ height $\times 20 \mathrm{~m}$ deep) of 2000 white dots was simulated. The dots were presented on a black background. For each frame (rate, $\sim 75$ $\mathrm{Hz}$ ), a step displacement for each anti-aliased dot (size, $10 \mathrm{~min}$ arc) was computed using the following formula for the velocity vector $\vec{p}_{i}$ associated with moving dot $i$ (Koenderink and van Doorn, 1987):

$$
\vec{p}_{i}=-\frac{T}{D_{i}}\left(\vec{t}-\left(\vec{d}_{i} \cdot \vec{t}\right) \vec{d}_{i}\right)-\vec{R} \times \vec{d}_{i}
$$

In this formula, $\vec{R}, \vec{t}$, and $T$ denote the ego-motion parameters. The vectors $\vec{R}$ and $\vec{t}$ denote the spin of eye and its translation relative to the environment. They are expressed in retinal coordinates. $T$ is the speed of the forward motion. These parameters are the same for all dots. Unit vector $\vec{d}_{i}$ indicates the retinal direction from which each moving dot is seen by the eye, and scalar $D_{i}$ is its distance (for additional details, see van den Berg et al., 2001). In the control condition, the visual stimulus always consisted of a stationary random dot pattern. In all cases, the subjects were required to fixate (pursue) a stationary (moving) small yellow marker on the screen for the duration of the trial.

All high-resolution functional scans lasted 224 s (56 volumes) starting with a control state and then alternating between test and control states. Epoch length was $24 \mathrm{~s}$, with the last epoch in each run being a $32 \mathrm{~s}$ control state.

Experiment 1. Single-cell recording studies in macaque area MST have described modulation of responses to optic flow by nonvisual signals related to eye and head movements (Bradley et al., 1996; Shenoy et al., 1999, 2002; Ilg et al., 2004). This modulation is believed to provide an important step toward coding of head-centric flow. In experiment 1 , we examined whether extra-retinal eye movement signals modulate opticflow-related BOLD responses in the human MT+ complex.

The visual stimulus in this experiment consisted of a radially expanding flow pattern that corresponds to forward motion at $T=4 \mathrm{~m} / \mathrm{s}$. This stimulus was viewed with a fixating eye or while the subject pursued a moving point target. In the fixation condition, both the yellow fixation point and focus of expansion remained fixed at the center of the screen. In the pursuit condition, we added the sinusoidal, horizontal motion of the target (four cycles of $1 / 6 \mathrm{~Hz}$; peak velocity of $6 \%$; peak amplitude of $\sim 6^{\circ}$ ) to each point of the flow display so as to keep the focus of expansion centered on the fovea. Thus, for pursuit eye movements with a gain of 1.0 (an assumption that we investigated in a control experiment), the flow pattern on the retina was the same for the fixation and the pursuit condition and contained no rotational components, i.e., the retinal rotational speed is 0 , but the head-centric rotational speed equals the pursuit speed. The retinal- and head-centric speed refers, in this paper, to the (global) rotational component of the flow field, not to the local motions that differ according to the combination of the rotation and the forward motion of the eye and the location of the visual field.

Data were collected from seven subjects. In each subject, we presented a total of eight fixation trials and eight pursuit trials across four successive runs.

Experiment 2. In this experiment, we probed the substructure of MT+ further by testing whether functional subdivisions of this region transform the retinal flow into head-centric flow. Our stimuli aimed to meet the following demands. (1) The head-centric flow should be controlled independently from the retino-centric flow. (2) The visual task should evoke motion judgments relative to the environment. Such a task presumably directs the subject's attention to signals that combine retinocentric flow with extra-retinal signals, promoting (e.g., head-centric flow) related fMRI signals. (3) The stimulus should allow for testing of a metric relation between fMRI signals and head-centric flow magnitude.

Figure $1 A$ shows the stimulus that fulfilled these requirements. The stimulus simulates a head rotation during forward translation of the body along an undulating path. Importantly, the simulation assumed that the gaze line was always aligned with the heading direction. One can think of this simulation as the flow that is received by a front-seat pas- 
A
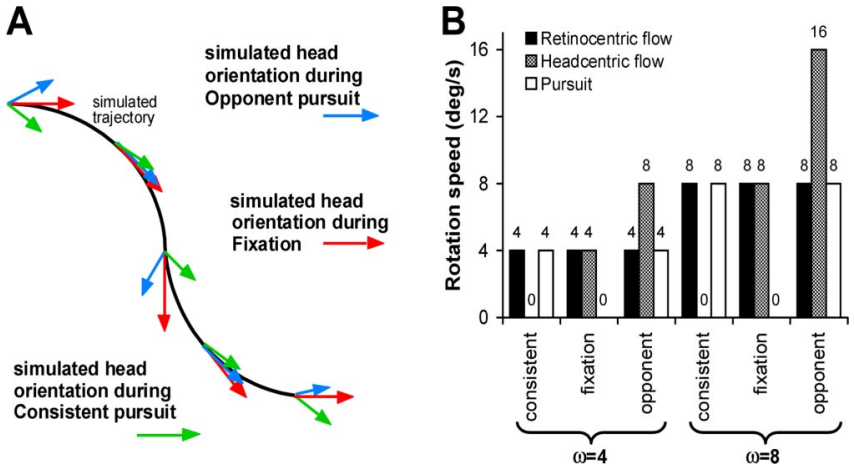

Figure 1. Stimuli in experiment 2.A, Top view of the simulated head-in-space rotations and undulating path of the subject. Arrows indicate the head orientation relative to the environment under the three experimental conditions. Note that the gaze line is always in the direction of the forward motion, which produces identical retinal flow patterns under the three conditions. For the flow fields presented on the screen, see supplemental movies (available at www. jneurosci.org as supplemental material). $\boldsymbol{B}$, Speed of the eye-in-head pursuit as well as retinaland head-centric rotational flow components under the consistent, opponent, and fixation condition for each of the two trajectories simulating rotations of the gaze line relative to the environment at $\omega=4^{\circ} / \mathrm{s}$ and $\omega=8^{\circ} / \mathrm{s}$, respectively.

senger of a car riding along a winding road while he fixates a mark on the windscreen. Even when the passenger maintains a fixed orientation of eye-in-head and head-on-body, his eyes are rotated relative to the environment as the car turns around each bend. If the passenger instead oscillates his head around a vertical body axis, this head rotation will carry the eyes away from the mark on the windscreen, unless he makes a compensatory eye rotation. In the latter case, the gaze line changes over time just as in the condition in which eye and head had a fixed orientation on the body. It is important to realize that, when the forward motion and the rotation of the gaze line remain the same, the retinal flow pattern remains the same as well, regardless of the head rotation. This holds because the gaze rotation and the translation completely specify the velocity of the eye relative to the environment (Koenderink, 1986). Thus, by keeping gaze aligned with the heading direction, the head-centric flow can be controlled independently from the retino-centric flow, which fulfills the first requirement.

Our visual stimuli simulated the flow induced by the undulating motion of the car and the head oscillations but not the eye-in-head rotation (supplemental movies, available at www.jneurosci.org as supplemental material). The frequency of the head rotation and that of the undulating path of the car were identical. In addition, we presented a yellow fixation point to simulate the mark on the windscreen that is always aligned with the direction of motion of the car. The subjects were instructed to fixate or pursue this yellow dot and attend to their direction of self-motion through the cloud of dots, supporting the second requirement. We point out that our stimuli did not aim to support the ego motion percepts that normally accompany head rotations (because there was no vestibular stimulation). Accordingly, our subjects did not have the illusion that they were rotating their head in the simulated head-rotation conditions. Rather the visual environment was seen as an approaching cloud of dots that appeared to oscillate relative to the head (and body), and the vantage point was seen to move along a sinusoidal trajectory.

We presented four cycles of oscillation in each trial of $24 \mathrm{~s}$. In the "fixation" condition, we merely simulated forward motion of the car along the sinusoidal path (forward speed, $4 \mathrm{~m} / \mathrm{s}$ ), i.e., the simulation assumes that the head is not rotating relative to the car (Fig. $1 \mathrm{~A}$, red arrows). The fixation point thus remained stationary at the center of the display. In the "opponent" condition, we simulated in addition to the forward motion a head rotation (Fig. $1 \mathrm{~A}$, blue arrows) that was in-phase with the undulations of the forward motion. Thus, when a rightward turn of the car was simulated, the simulated head rotation was also rightward. This means that, to fixate the yellow marker on the windscreen of the car, the eye has to turn leftward in the head. Because the head rotation and the movement of the car were simulated, the yellow fixation point moved leftward on the display, i.e., eye pursuit was opponent to the simulated car (= body) rotation. In the "consistent" condition, we simulated in addition to the forward motion a head rotation (Fig. $1 \mathrm{~A}$, green arrows) that was in anti-phase with the undulations of the forward motion. Now the yellow marker on the display rotated in the same direction as the simulated car (= body) motion.

If the subject pursues the yellow target accurately (gain of $\sim 1.0$, which we verified in a control experiment), the oscillation of the gaze line relative to the cloud of dots remains the same for all three conditions. However, the simulated rotation of the head relative to the environment is different. In the consistent condition, the orientation of the head relative to cloud of dots remains the same throughout the simulation (Fig. $1 \mathrm{~A}$, green arrows), i.e., the head-in-space rotation amplitude is 0 . There is only forward and sideways translation of the head. In the fixation condition (Fig. $1 A$, red arrows), the orientation of the head varies relative to the cloud of dots just as that of the car, and, in the opponent condition, the head rotates relative to the environment by twice the amount of the car rotation. Thus, for identical retinal flow patterns, these three conditions present three levels of head-centric rotational flow: 0 (consistent), $\omega$ (fixation), and $2 \omega$ (opponent). This supports the third requirement.

Data were collected from six subjects (10-16 runs per subject, four test trials, and five control epochs per run). In each subject, we presented two levels of $\omega: 4$ and $8 \%$ s (Fig. 1 B). These numbers represent the peak velocity of the simulated head rotation relative to the car. The oscillation frequency $(f)$ was $1 / 6 \mathrm{~Hz}$. The peak amplitudes of simulated head oscillations relative to the car and the undulation of the path of the car relative to the environment were given by $\omega /(2 \pi f)$. In total, four levels of headcentric rotational flow were investigated ( $h f=0,4,8$, and $16 \%$ s). Note that the $8 \%$ s head-centric flow condition was presented for two levels of $\omega$ : fixation with $\omega=8 \% \mathrm{~s}$ and opponent with $\omega=4 \%$. Note in addition that the speed of rotation in the retinal flow was doubled in the $\omega=8 \% \mathrm{~s}$ condition compared with the $\omega=4 \%$ condition. Thus, also the $0 \% \mathrm{~s}$ head-centric flow condition was presented for two levels of $\omega$ : consistent with $\omega=4 \%$ and consistent with $\omega=8 \%$ s.

Control experiment. Our choice of the target motions in experiments 1 and 2 promotes accurate pursuit (Collewijn and Tamminga, 1984, 1986). To verify that the subjects indeed performed correctly in the fixation and pursuit tasks, we did a control experiment outside the MRI scanner in which we measured the eye movements of five subjects with the scleral search-coil technique [resolution, $<0.05^{\circ}$; noise, $<0.1^{\circ}$ peak-to-peak; sampling rate, $500 \mathrm{~Hz}$ (Collewijn et al., 1975)]. This experiment was done in a mock scanner to ensure that the test stimuli were viewed in the same way as in the MRI experiments (see above, Visual stimuli).

Data analysis. Image analysis was performed using Brain Voyager 2000 (version 4.8; Brain Innovation, Maastricht, The Netherlands). Surface coil images were manually realigned to the head-coil images, and the images were spatially normalized according to the atlas of Talairach and Tournoux (1988) to obtain standardized coordinates for the regions of interest. Anatomical images from each subject were then segmented at the gray/white matter boundary and subsequently inflated and flattened for visualization purposes (Goebel et al., 1998).

Functional data underwent 3D motion correction, and the time courses within each voxel were corrected for linear drift. We excluded any ( 2 of 128) scans in which motion artifacts were observed in a cinematic loop. After this preprocessing, functional runs within a subject were analyzed with a voxel-by-voxel regression analysis.

To quantify the amplitudes of the BOLD responses, we used the general linear model (GLM) with a predictor for each experimental condition. Predictors were entered as reference functions reflecting the temporal sequence of experimental and control conditions convolved with the hemodynamic response (lag values of 1 corresponding to a $4 \mathrm{~s}$ delay). The regression statistics were corrected for temporal autocorrelation of the BOLD signal.

To generate functional maps, we computed the conjunction of two GLM contrasts. The first contrast identified voxels that were more active in the motion conditions compared with the static control condition. The second contrast compared the activation between different motion conditions (see Results). The threshold for each contrast was set at $p<$ 0.05 . By only considering clusters of three or more contiguous voxels, the 


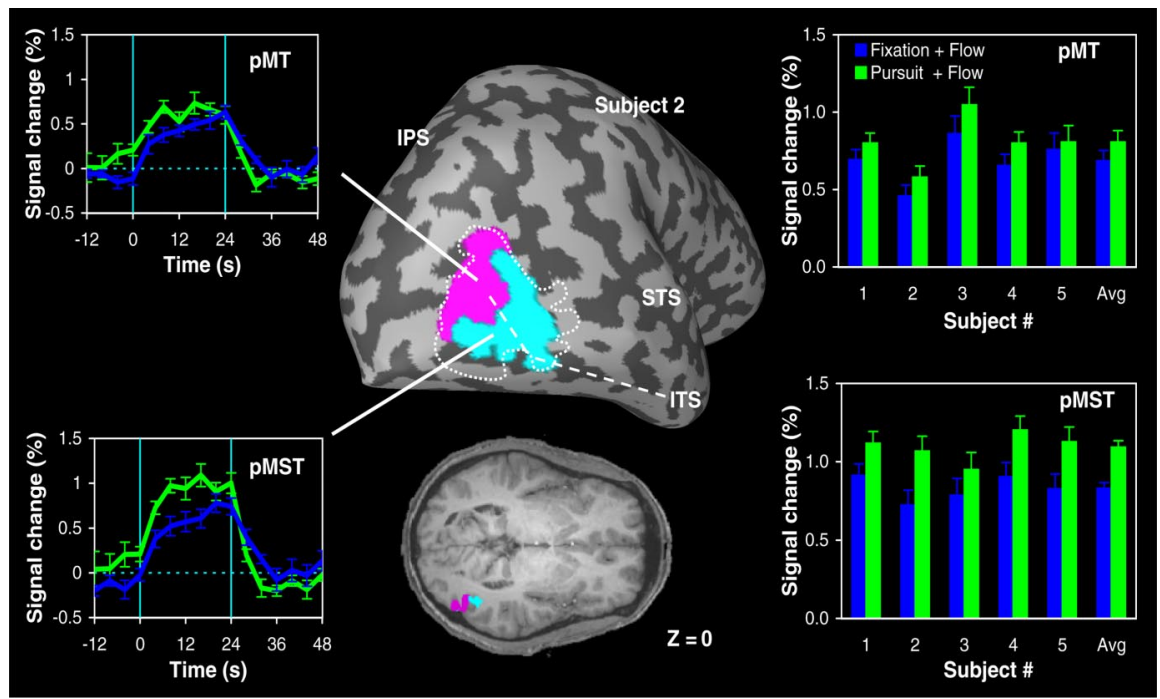

Figure 2. Influence of pursuit eye movements on $\mathrm{fMRI}$ activity in putative area MT and MST. Left, Region-of-interest analysis of the $B 0 L D$ responses in MT + for subject 2. The MT + cluster was functionally subdivided into two areas that we tentatively identify as homologs of macaque area MT and MST (Dukelow et al., 2001) (see Materials and Methods). The anatomic location of area pMT (magenta) and area PMST (turquoise) in subject 2 is indicated on the inflated brain and axial slice at $z=0$. The dashed line indicates the outline of MT + . Time courses show the BOLD signal in each ROI for expanding optic flow with the focus of expansion centered on the fovea during fixation (blue) and pursuit (green) relative to the response of the area to stationary dots. Signals are aligned with the onset of the motion stimulus and averaged across trials (error bars indicate \pm 1 SEM). Vertical line segments indicate the onset and offset moments of the 24 s motion epoch. Right, Magnitude of the signal changes in area pMT and pMST under the two experimental motion conditions for subjects 1-5. Avg, Averaged response across all five subjects. Error bars indicate SEM. ITS, Inferior temporal sulcus; STS, superior temporal sulcus.

effective $p$ value was 0.005 (Forman et al., 1995). Maps were superimposed on T1-weighted anatomical reference scans.

Localization of MT and MST. We localized the MT+ cluster (i.e., area MT plus other adjacent motion-sensitive areas, including MST) in the dorsal/posterior limb of the inferior temporal sulcus (Zeki et al., 1991; Watson et al., 1993; Tootell et al., 1995a,b; Dumoulin et al., 2000) using a wide-field optic flow minus static comparison. The dissociation between the putative human homologs of macaque areas MT and MST was based on the notion that, in macaques, MT receptive fields primarily encode the contralateral visual field, whereas receptive fields in MST dorsal are much wider and extend well into the ipsilateral visual field (Dukelow et al., 2001; Huk et al., 2002).

We used the data from a previous fMRI study (Dukelow et al., 2001) to distinguish putative area MT (pMT) from putative area MST (pMST) in seven subjects (1-7). For the other three subjects in this study, we did not distinguish between MT and pMST subdivisions. The motion stimulus in that study was a radially expanding dot pattern (200 dots; average speed, $8.0^{\circ} \mathrm{s}$; white dots on a black background) with the focus of expansion located at the center of the display. However, no dots were displayed in the central $30^{\circ}$ of the display. Subjects fixated at the center while dots appeared from 15 to $45^{\circ}$ in the periphery in either the contralateral (left) or ipsilateral (right) visual field. The control condition consisted of an identical display, but the dots were stationary. Data were analyzed using the general linear model, with response to contralateral motion being one predictor and response to ipsilateral motion as a second predictor.

For each individual subject, a region of interest (ROI) was generated for pMT based on contiguous voxels in MT + that were activated significantly by motion in the contralateral visual field compared with stationary but not activated significantly by motion in the ipsilateral visual field. ROIs were generated for pMST based on contiguous voxels that were activated significantly by motion in the ipsilateral visual field compared with stationary. Because the contralateral/ipsilateral visual stimuli did not cover the central visual field ( $\pm 15^{\circ}$ from the fovea), the foveal representation of area MT may not have been included in the ROIs generated for pMT. Conversely, a recent fMRI study with macaque monkeys (Orban et al., 2003) indicated that visual motion stimuli also produce significant BOLD responses in the fundus of the superior temporal sulcus
(FST). The ROIs generated for pMT and pMST may therefore include subdivisions of a region that is homologous to motion area FST in the monkey.

\section{Results}

In all 10 subjects that participated in the experiments, motion stimuli produced robust activation (compared with stationary) in the dorsal/posterior limb of the inferior temporal sulcus. The location of this activation [Talairach coordinates $(x, y, z)$ of $(43,-67,3)$ ] was consistent with previous reports of human $\mathrm{MT}+$ (Zeki et al., 1991; Watson et al., 1993; Dupont et al., 1994; McCarthy et al., 1995; Tootell et al., 1995a,b; Smith et al., 1998; Sunaert et al., 1999; Dumoulin et al., 2000; Dukelow et al., 2001). Additionally, optic-flowrelated functional activity was observed in the intraparietal sulcus (IPS), the calcarine sulcus (likely corresponding to the primary visual cortex, area V1), the parietooccipital sulcus (POS), and in the collateral sulcus.

\section{Experiment 1}

Single-cell recording studies have described modulation of responses to optic flow by nonvisual signals related to eye position, eye movement, and head movement in area MST of macaques (Bradley et al., 1996; Bremmer et al., 1997; Shenoy et al., 1999, 2002; Upadhyay et al., 2000; Ilg et al., 2004). This modulation is believed to provide an important step toward compensation for the effects of ego-motion on the retinal flow patterns. Previous fMRI studies have reported robust activation in most of the human MT+ complex during pursuit of a small moving target (Barton et al., 1996; Petit and Haxby, 1999; Dukelow et al. 2001), but it is not known whether pursuit modulates responses to optic flow. In experiment 1 , we therefore examined the influence of pursuit eye movements on the BOLD responses in the $\mathrm{MT}+$ cluster (i.e., area MT plus other adjacent motion-sensitive areas, including MST) during a radially expanding flow pattern with the focus of expansion centered on the fovea.

As shown in Figure 2, we first applied a region-of-interest analysis, which allowed us to dissociate between the responses of two functional subdivisions of MT + that we tentatively identify as homologs of macaque area MT and MST. To illustrate this analysis, the left panels in Figure 2 depict the anatomic location of pMT (magenta) and pMST (turquoise) in one of our subjects as defined by our functional MT-MST localization protocol (Dukelow et al., 2001) (see Materials and Methods), as well as the averaged signal time courses from each of these ROIs during the fixation and pursuit conditions. Note that, in this subject, the pursuit task produced an increase in the activation of area pMST, whereas the signal changes in area pMT were approximately the same when the motion stimulus was viewed with a fixating eye or when the subject pursued a moving target. To quantify the amplitudes of these signal changes, we used a GLM with responses to flow during fixation being one predictor and responses to flow during pursuit as a second predictor.

The right panels in Figure 2 summarize the results of this procedure for the five subjects in experiment 1 for which MT- 
MST dissociation data were available. Interestingly, we observed that the opticflow-related activation of area pMST was consistently enhanced by the pursuit task and that these changes in activity were statistically significant in all but one subject (GLM analysis, $p<0.05$ ). The mean \pm SD difference between the activation in the fixation task and the pursuit task was $0.27 \pm 0.07 \%$ of the baseline. A paired Student's $t$ test across subjects indicated a similar but smaller effect in pMT $(p<0.01$; mean difference, $0.12 \pm 0.05 \%)$. For the individual subjects, the enhancement produced by the pursuit task in pMT was in fact not statistically significant (GLM analysis, $p>0.1$ in all five subjects).

To further examine the functional subdivision of $\mathrm{MT}+$, we also performed a voxel-by-voxel GLM analysis on the fMRI data from all seven subjects that participated in experiment 1 . In each subject, we observed two subregions of voxels within $\mathrm{MT}+$ that showed a significantly different response in the pursuit condition compared with the fixation condition (GLM contrast, $p<0.05$ ). Table 1 lists the Talairach coordinates of these clusters. As illustrated in Figure 3 for one of our subjects (same subject as in Fig. 2), the responses in both ROIs were strongly enhanced by the pursuit task. Interestingly, and in line with the results from Figure 2, one of the ROIs (yellow) showed considerable overlap with area pMST. As shown in the flat maps of Figure 4, overlap of this ROI with area pMST was consistently observed for all five subjects in experiment 1 for whom we had pMST and pMT localization data. The second ROI (red) was typically found more lateral, slightly posterior, and somewhat inferior to the first ROI and showed practically no overlap with area pMST (Figs. 3, 4). We will refer to these ROIs as the medial (yellow) and lateral (red) ROI, respectively, because a comparison of their Talairach coordinates (Table 1) with paired Student's $t$ tests indicated that the second ROI was more lateral than the first $(p<0.05)$. The $y$ and $z$ coordinates were not significantly different. Both the medial and lateral ROI showed little or no overlap with area pMT (Figs. 3, 4).

The average volume of the medial and lateral pursuit ROI across all seven subjects was $0.3 \pm 0.1 \mathrm{~cm}^{3}$ (mean $\pm \mathrm{SD}$; median, $0.3 \mathrm{~cm}^{3}$ ) and $0.5 \pm 0.3 \mathrm{~cm}^{3}$ (median, $0.4 \mathrm{~cm}^{3}$ ), respectively. To estimate the amount of overlap of these ROIs with area pMT and area pMST, we used the MT-MST dissociation data that were available for subjects $1-5$. In these subjects, we observed that $56 \pm$ $15 \%$ of the voxels of the medial pursuit ROI overlapped the pMST region, whereas only $14 \pm 10 \%$ of the voxels of this ROI fell within the pMT region. For the lateral pursuit ROI, we found that only $9 \pm 12$ and $15 \pm 9 \%$ of the voxels fell within the pMST and pMT region, respectively.

Figure 5 compares the amplitudes of the BOLD responses in the pursuit and fixation condition for the lateral and medial pursuit-modulated ROIs in all seven subjects. Note the robust modulation of the responses by the pursuit task in both ROIs for all subjects. Interestingly, however, the responses of the lateral $\mathrm{ROI}$ in the fixation condition were much weaker compared with the ones in the medial ROI. In five subjects, the responses of the lateral ROI during fixation were in fact not significantly different from 0 (GLM analysis, $p>0.3$ ). Averaged across all subjects, the lateral ROI showed no significant response to the expanding flow pattern in the fixation condition (paired $t$ test, $p=0.07$ ).

In all seven subjects, we also noticed pursuit-related enhancement of the BOLD responses in subregions along the POS (medial, bilateral activation at $z=0$ in Fig. 3) and the IPS. We have not analyzed these responses in quantitative detail. In contrast, flowrelated activity in voxels along the calcarine sulcus was not significantly modulated by the pursuit task (see functional map at $z=0$ in Fig. 3). The latter may not be surprising because this task produced nearly identical retinal flow patterns with the focus of expansion centered on the fovea (see below, Control experiment).

\section{Experiment 2}

The first experiment shows that the pursuit task boosts the BOLD response to visual flow in $\mathrm{MT}+$. Such a modulation by pursuit could be attributable to BOLD responses to independent pursuit and flow signals. The second experiment aims to show that true 


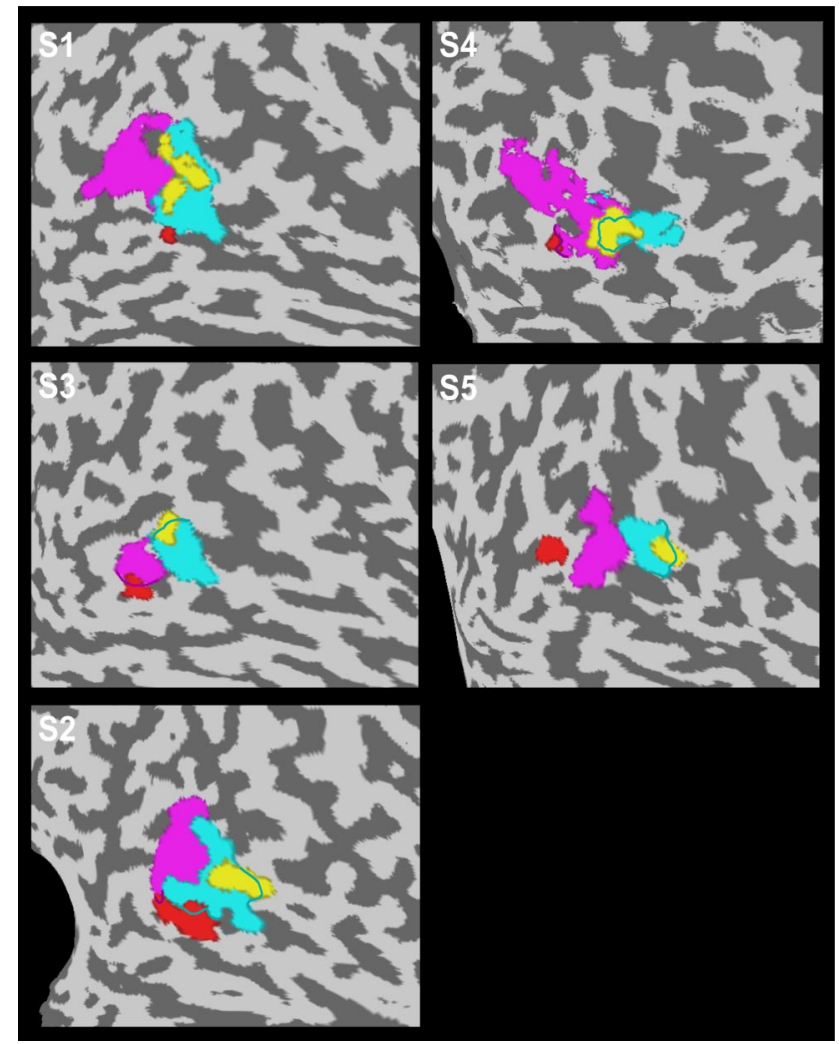

Figure 4. Localization of MT + subregions with pursuit-enhanced responses in five different subjects. Flattened surface maps of the brains of all five subjects (S1-S5) in experiment 1 with identified pMT (magenta) and pMST (turquoise) subdivisions. Each flat map shows the location of the two subregions (yellow and red) in which the pursuit task produced enhanced responses to expanding optic flow. Note that one of the pursuit-modulated ROls (yellow) showed considerable overlap with area PMST in all five subjects.
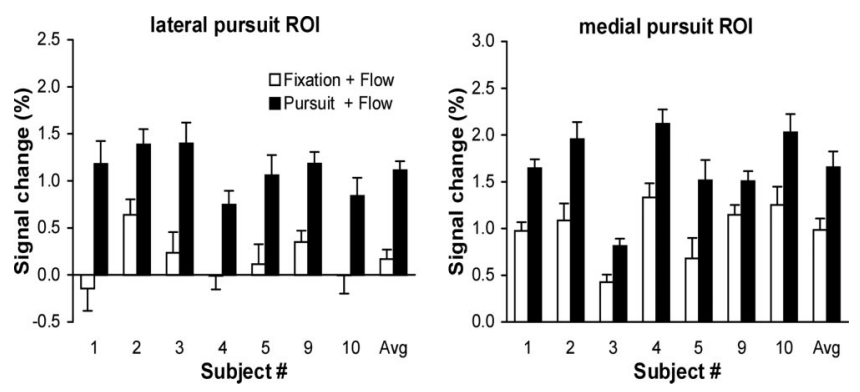

Figure 5. Pursuit-modulated fMRI responses in two MT+ subregions. Percentage signal change in the medial and lateral MT + subregion for expanding optic flow with the focus of expansion centered on the fovea during fixation $(\square)$ and pursuit $(\square$ ) relative to the response of the ROI to static dots. Data from the seven individual subjects are presented on the left, and the average (Avg) data are presented on the right. Error bars indicate SEM.

interaction occurs between pursuit and flow signals within MT+ that may reflect the computation of head-centric flow.

To test whether such interactions exist within the MT+ complex without making specific assumptions regarding the nature of these interactions, we compared the fMRI activity in the consistent and opponent condition. As explained in Materials and Methods, both the pursuit movements and the retinal flow patterns were the same under these conditions. Only the phase relation between pursuit and the retinal rotational flow component was opposite, resulting in head-centric rotational flow that was either 0 (consistent) or $2 \omega^{\circ} / \mathrm{s}$ (opponent). Because the BOLD
Table 2. Talairach coordinates of the pHFR for all six subjects participating in experiment 2

\begin{tabular}{llll}
\hline Subject & $x$ & $y$ & $z$ \\
\hline 1 & 44 & -68 & 2 \\
2 & 38 & -61 & 0 \\
3 & 43 & -63 & 0 \\
6 & 43 & -59 & 6 \\
7 & 49 & -61 & 1 \\
8 & 41 & -64 & 7 \\
Mean \pm SD & $43 \pm 4$ & $-63 \pm 3$ & $3 \pm 3$ \\
\hline
\end{tabular}

signal integrates the responses on a long timescale, independent flow and pursuit responses would yield a BOLD signal that only depends on the amplitudes of the pursuit and the rotational flow signals. In contrast, if the BOLD signal reflects a neural interaction representing head-centric flow, the response should also depend on the phase relation between pursuit and rotational flow. Thus, the activation will be different for consistent and opponent pursuit conditions in regions that show interactions between pursuit- and flow-related signals, whereas regions carrying only independent visual and nonvisual signals will exhibit no change.

Functional data were analyzed with a voxel-by-voxel GLM analysis. In all six subjects that participated in this experiment, we observed an ROI within MT + that was more active in the opponent conditions than in the consistent conditions as determined by a GLM contrast between these conditions $(p<0.05)$. For Talairach coordinates, see Table 2. Figure 6 illustrates this finding for subject 2 (same subject as in Figs. 2, 3). Note that the putative head-centric flow region ( $\mathrm{pHFR}$, yellow) was found within the anterior portion of the MT+ complex, close to the border of area pMST (turquoise) and area pMT (magenta), but mostly overlapping area pMST. As shown in the time courses of Figure 6, the response of this ROI to the optic flow stimulus was strongly enhanced in the opponent condition (blue) compared with the consistent condition (green) at both levels of $\omega$ (thin-dark, $4 \%$; thick-bright, $8^{\circ} / \mathrm{s}$ ). These differences cannot be attributable to differences in the retinal or extra-retinal signals per se because, for each level of $\omega$, both the pursuit and the retinal flow patterns were the same (see Materials and Methods); it was the different phase relationship between pursuit and retinal flow that caused the difference in ROI response. Furthermore, this response property was specific to this subregion of the MT + complex because it was not reflected in the mass activity of pMT and pMST.

The average volume of the pHFR across all six subjects was $0.5 \pm 0.5 \mathrm{~cm}^{3}$ (mean $\pm \mathrm{SD}$; median, $0.3 \mathrm{~cm}^{3}$ ). In line with its anteromedial location in the MT + complex, a considerable overlap with the pMST region was observed in the five subjects (1-3, 6 , and 7) with MT/MST localizer data. On average, $62 \pm 23 \%$ of the voxels overlapped the pMST region, whereas only $14 \pm 18 \%$ of the voxels were found within the pMT region. The overlap with pMST in each of these subjects is further illustrated in the flat maps of Figure 7, which show the anatomical localization of the pHFR with respect to the subjects' $\mathrm{pMT}$ and pMST regions. The bottom panels in Figure 7 present the results of two experiments with subject 2. Although the first session (right panel) was a pilot experiment in which we applied only one level of $\omega(6 \%$; corresponding to head-centric rotational flow of $h f=0,6$, and $12 \%$ ), we include these data to demonstrate that sensitivity to headcentric flow was reproducibly observed within the same region of pMST.

In each subject, we further quantified the amplitude of the BOLD responses in the pHFR for all six test conditions (i.e., 
fixation, consistent, and opponent at two levels of $\omega$ ) by fitting a general linear model to the ROI time course data using a predictor for each test condition. As explained in Materials and Methods (compare with Fig. 1), the six test conditions involved two different levels of retinocentric rotational flow speed ( 4 and $8 \%$ ) and four different head-centric rotational flow speeds $(0,4,8$, and $16 \%$ s). If the observed interaction between pursuit and retinal rotational flow indeed reflects the presence of a head-centric rotational flow signal, there could also be metric relation between the BOLD responses and the head-centric speed of the rotational flow component.

To explore this possibility, we plotted the amplitudes of the BOLD responses as function of the retino-centric (Fig. $8 A$ ) and head-centric (Fig. $8 B$ ) speed of the rotational flow. For clarity, the data from each individual subject were normalized with respect to the mean ampli-

tude of the signal changes under all test conditions. Note that the data in Figure $8 B$ show a robust increase in the activation as function of head-centric speed in all six subjects (mean \pm SD correlation, $r=0.79 \pm 0.14)$. In contrast, the data in Figure $8 \mathrm{~A}$ scatter around $100 \%$ for both retinal speed levels, indicating that the responses were not systematically influenced by the retino-centric speed of the rotational flow component. Only in the fixation conditions, i.e., when the retinocentric and head-centric rotational flow speeds were the same, did we observe a consistently bigger response for the $\omega=8 \% \mathrm{~s}$ condition compared with the $\omega=4 \%$ s condition.

Additional inspection of the data suggested, however, that head-centric flow was not the only factor determining the BOLD responses. For example, the $8 \%$ s head-centric flow condition was presented for two levels of $\omega$ (Fig. $1 \mathrm{~B}$, fixation with $\omega=8 \%$ and opponent with $\omega=4 \%$ ), but, on average, the responses were significantly lower in the $\omega=8 \%$ fixation condition compared with the $\omega=4 \%$ s opponent condition (paired $t$ test, $p<0.05$ ). To quantify the contributions of the different visual and nonvisual factors, we therefore modeled the ROI time course data, $Y(t)$, as function of retino-centric flow, head-centric flow, and pursuit. The multiple linear regression model used in this analysis was given by the following:

$$
\begin{array}{r}
Y(t)=\beta_{1} \times F c(t)+\beta_{2} \times R s(t)+\beta_{3} \times H s(t)+ \\
\beta_{4} \times P b(t)+\beta_{5} \times P s(t)+\beta_{6}
\end{array}
$$

The first term in this model, $F \mathcal{C}$, represents a constant signal change in the motion conditions compared with the static control condition. The second term, Rs, models the effect of presenting two levels of retino-centric rotational flow. The third term, $H s$, relates the signal changes to the head-centric flow, assuming that the responses are linearly related to the peak velocity of the rotational flow component. The last two terms model a response bias attributable to pursuit eye movements, $P b$, and the effect of presenting two levels of pursuit speed, $P s$, respectively. The constant $\beta_{6}$ signifies the baseline activity in the stationary control condition.

Figure 9 summarizes the results of this factorial regression

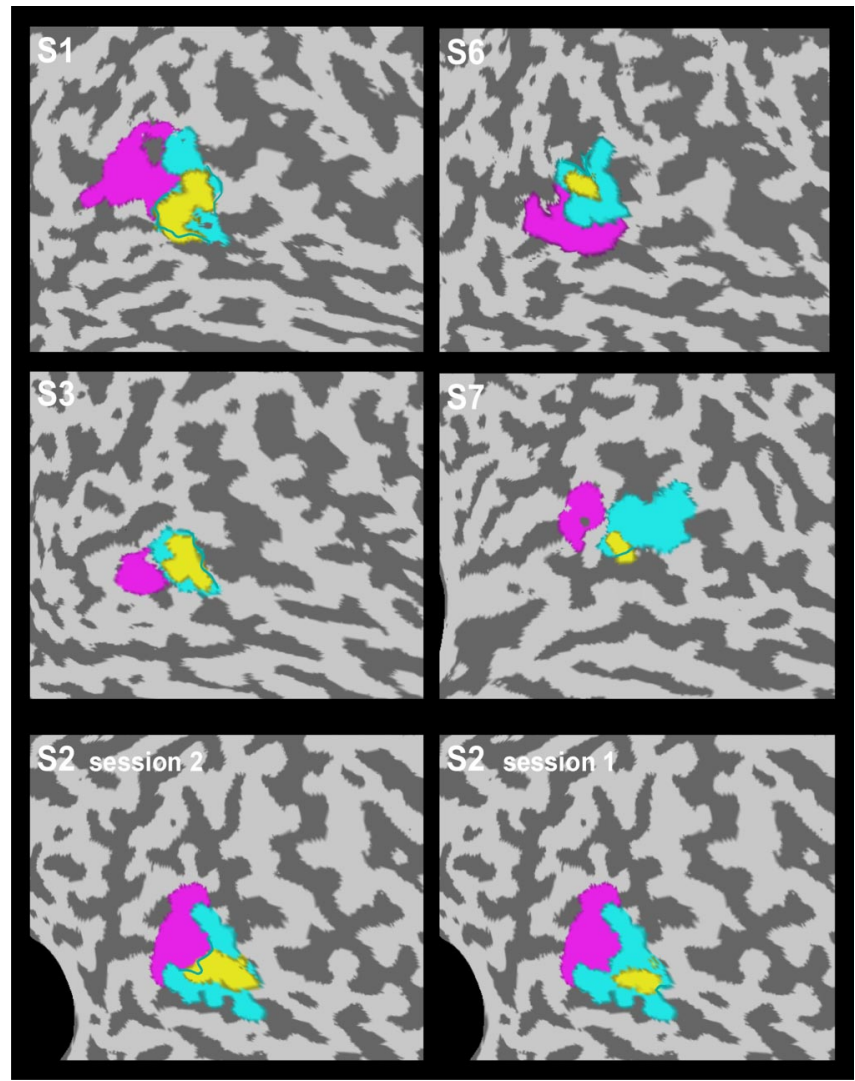

Figure 7. Localization of the pHFR in five different subjects. Flat maps of the brains of five subjects $(S 1-S 3, S 6,57)$ showing the location of the pHFR (yellow) relative to the subjects' identified pMT (magenta) and pMST (turquoise) subdivisions. Note that the pHFR showed considerable overlap with area pMST in all five subjects. Bottom panels show the reproducibility of this finding for two experiments with subject 2 .

analysis. As expected, we observed a robust contribution of the constant optic-flow term in all six subjects (i.e., $\beta_{1}>0$; GLM contrast, $p<0.05$ ) (Fig. 9A). The amplitude of this response component was $0.90 \pm 0.19 \%$ of the baseline (mean $\pm S D$ ). In 

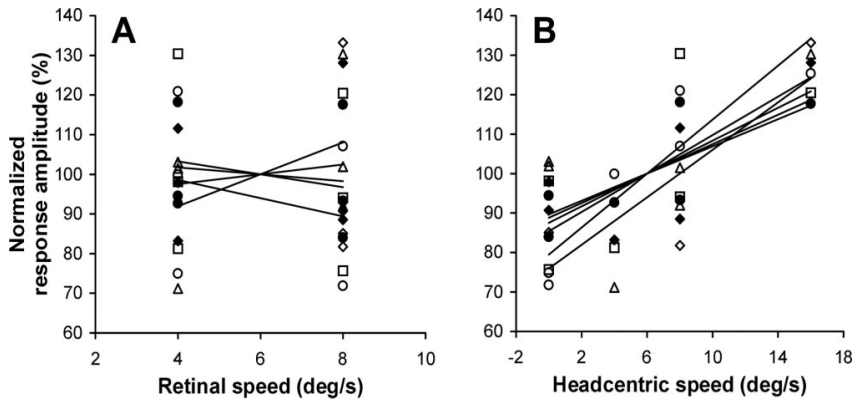

Figure 8. Response amplitudes of the pHFR correlate with the head-centric speed of rotational flow. Normalized response amplitudes plotted as function of retino-centric $(\boldsymbol{A})$ and headcentric $(\boldsymbol{B})$ rotational flow speed. Different symbols represent data from different subjects. $100 \%$ corresponds to the mean amplitude of BOLD responses across all motion conditions. Mean \pm SD slopes of the regression lines (solid) in $\boldsymbol{A}$ and $\boldsymbol{B}$ were $0.6 \pm 2.0 \% \cdot \mathrm{deg}^{-1} \cdot \mathrm{s}^{-1}$ (not significantly different from 0 ) and $2.4 \pm 0.6 \% \cdot \mathrm{deg}^{-1} \cdot \mathrm{s}^{-1}(t$ test, $p<0.05)$, respectively.

line with the data in Figure $8 B$, the analysis also indicated that the BOLD responses were significantly modulated by the headcentric speed of the rotational flow in all six subjects (i.e., $\beta_{3}>0$; GLM contrast, $p<0.05$ ) (Fig. $9 C$ ). The partial correlation between this regression variable and the BOLD signal ranged between 0.63 and 0.83 (mean $\pm \mathrm{SD}, 0.71 \pm 0.07$ ), and the average gain across subjects was $\beta_{3}=0.33 \pm 0.13 \%$ of the baseline per degree per second. In contrast, we observed no statistically significant influence of retino-centric rotational flow speed (i.e., $\beta_{2}=$ $0)$ (Fig. 9A), neither within subjects (GLM contrast, $p>0.05$ ) nor across subjects (paired $t$ test, $p>0.05$ ). There was, however, a significant influence of pursuit eye movements (Fig. 9B). That is, pursuit by itself produced a systematic increase of the BOLD responses in the pursuit conditions (i.e., pursuit bias $\beta_{4}>0$ ), as was observed also in experiment 1 , but the influence of pursuit was not significantly different between the 4 and $8 \%$ s pursuit conditions (i.e., $\beta_{5}=0$ ).

\section{Control experiment}

Our experiments required that the subjects performed accurate pursuit. To demonstrate that our stimuli indeed induce accurate pursuit, Figure 10 shows the results of an off-line control experiment in which we measured the eye movements of five subjects in the pursuit and fixation tasks. Each data trace (black) represents the eye movements of an individual subject on a representative $24 \mathrm{~s}$ trial. Note that all subjects tracked the target (gray traces) quite accurately; the gains of the eye movement responses were close to 1.0 , and phase errors were practically 0 under all pursuit conditions. In the fixation trials, subjects typically maintained fixation within approximately $\pm 0.5^{\circ}$ of the stationary target.

Figure 10 bolsters the conclusion that the translation and rotation components of the retinal flow are virtually identical for the fixation and pursuit conditions. However, we did not adjust the display window during pursuit. The retinal location of the borders of the window therefore shifted during the pursuit tasks by twice the amplitude of the target movement $\left(6^{\circ}\right.$ in experiment $1 ; 8$ and $16^{\circ}$ in experiment 2) but not during the fixation task. These shifts were $<20 \%$ of the display width, but they cause a portion of high-speed retinal flow to shift in-and-out of view at retinal eccentricities $>35^{\circ}$. Could these shifts explain our findings?

Clearly, the change in visibility of motion vectors at the edges cannot explain the marked difference between the BOLD re-
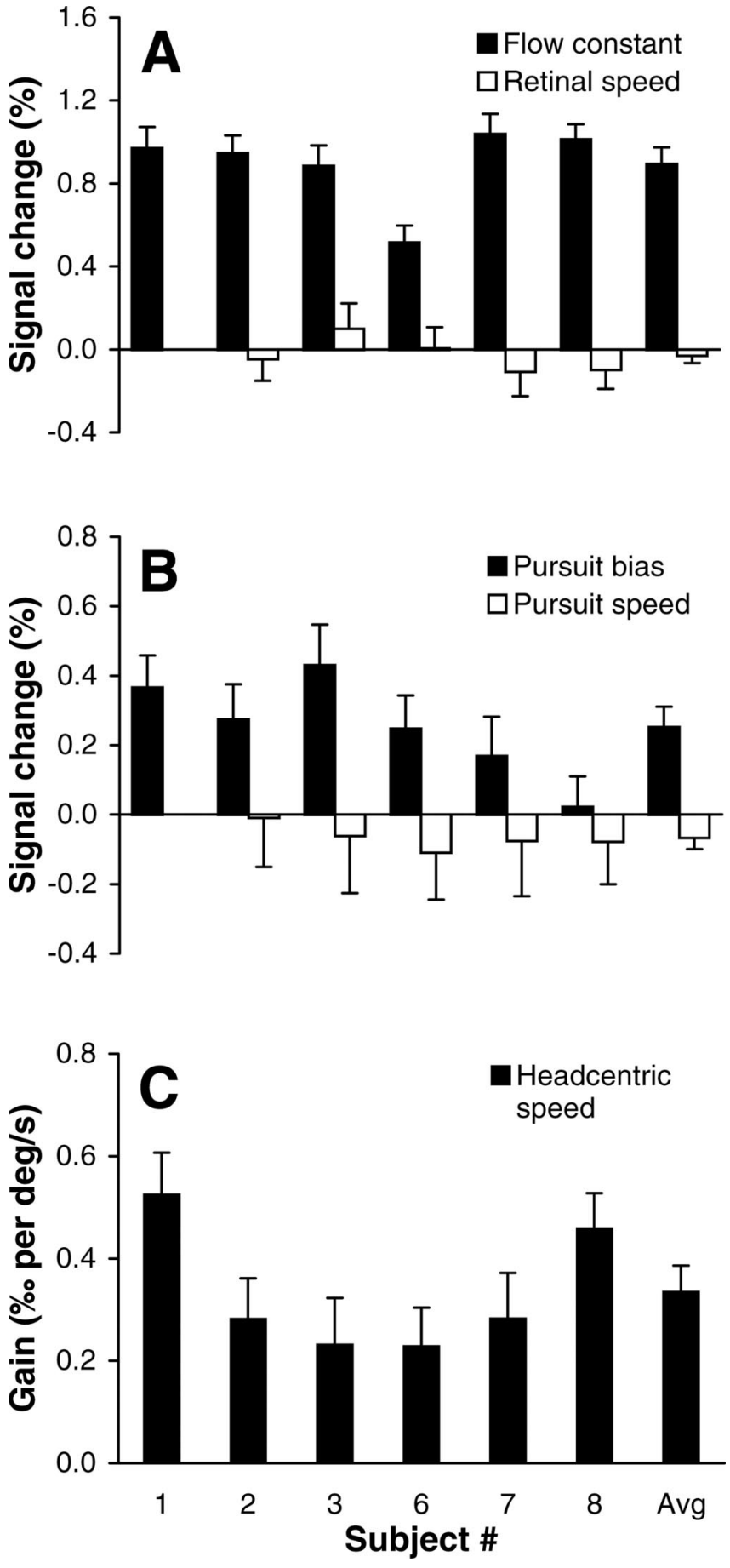

Figure 9. Model-based regression analysis. $A$, Percentage signal change attributable to the constant optic flow factor $\left(\beta_{1} ; \square\right)$ and the retino-centric rotational speed factor $\left(\beta_{2} ; \square\right)$. $\boldsymbol{B}$, Percentage signal change attributable to the pursuit bias $\left(\beta_{4} ; \square\right)$ and the pursuit speed factor $\left(\beta_{5} ; \square\right)$. C, Gain $\left(\beta_{3}\right)$ of the relation between head-centric rotational flow speed and the BOLD signal changes. Note scaling differences between panels. Error bars indicate SEM. Avg, Averaged values across all subjects.

sponses to opponent and consistent pursuit because, for either pursuit condition, the same portion of the retinal flow field shifts in-and-out of view across one cycle. This holds for both levels of $\omega(4$ and $8 \%$ s). Moreover, if edge effects are an important factor in our experiment that can explain the boosting of BOLD signals in the pursuit task compared with fixation, the responses should also depend strongly on the amplitude of the pursuit movements 


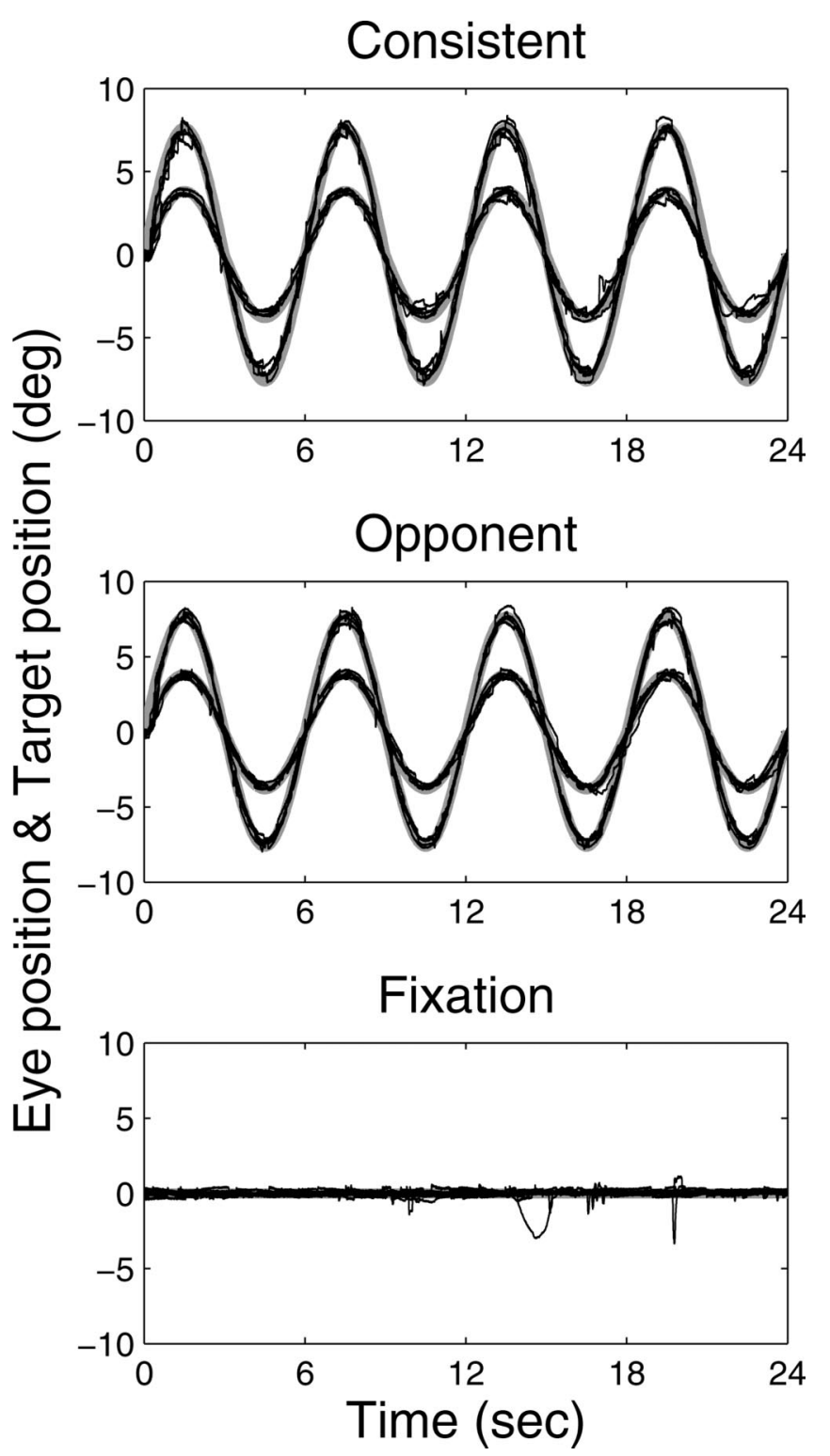

Figure 10. Eye movements of five subjects in the pursuit and fixation tasks. Eye movements were recorded during sinusoidal pursuit of a target at two different speeds (4 and $8 \%$ s peak velocity) in the consistent and opponent condition and during straight-ahead fixation. Eye and target movements were horizontal. Each black trace represents the eye movement data from an individual subject on a representative $24 \mathrm{~s}$ trial. Note that the subjects' overlapping data traces are closely aligned with the target position (thick gray) under each condition.

because that determines the displacement of the edges of the window on the peripheral retina. However, as illustrated in Figure 6 for one of our subjects (subject 2), this was not observed in the pHFR, in pMST, or in pMT. Note, for example, that 4 and $8^{\circ}$ consistent pursuit (for which head-centric rotational flow speeds are 0 ) produced in each of these ROIs the same BOLD response (green time courses), although the edge displacement is for $8^{\circ}$ consistent pursuit twice as large as for $4^{\circ}$ consistent pursuit. Also, the regression analysis in Figure 9 showed that there was no significant influence of pursuit amplitude in the subjects' pHFR (i.e., $\beta_{5}=0$ ).

As an additional control, we scrutinized the responses of area pMT in experiment 2 (Fig. 11). In line with experiment 1, pMT activity was enhanced in some pursuit conditions, i.e., the (averaged) responses during $4^{\circ}$ consistent pursuit and $8^{\circ}$ opponent
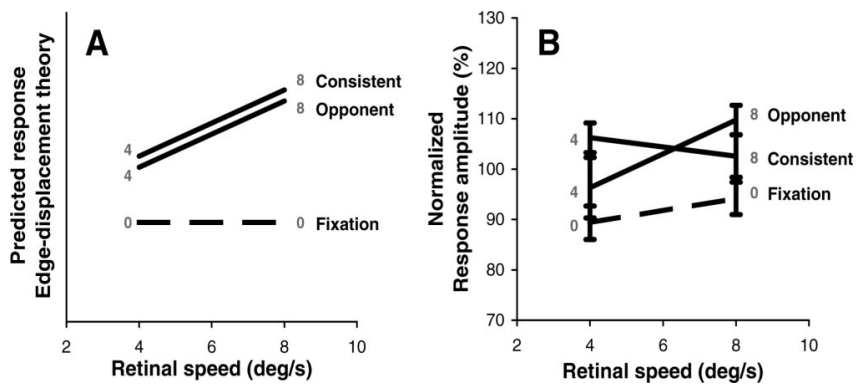

Figure 11. Activity in pMT not systematically influenced by edge displacement amplitude. $A$, Predicted responses of pMT under the six different test conditions in experiment 2 if the enhancement of BOLD signals is attributable to displacement of the stimulus window on the peripheral retina. Ordinate scaling is arbitrary. $\boldsymbol{B}$, Measured BOLD responses in area pMT. Data were normalized (as in Fig. 8) and averaged across five subjects. Error bars indicate SEM. Gray values indicate pursuit amplitudes (in degrees).

pursuit were significantly increased compared with fixation $(t$ test, $p<0.05)$. However, more importantly, the responses in these two different pursuit conditions, which involved peak-topeak edge displacements of 8 and $16^{\circ}$, respectively, were not significantly different when compared with one another! In fact, for most stimulus pairs with different edge displacements across the peripheral retina, the responses in pMT were indistinguishable, although the ROIs for pMT were obtained by stimulating the MT region that represents the (left) peripheral visual field (see Materials and Methods). Because MT probably is the major source of retinal motion signals for MST and other motion-sensitive areas in $\mathrm{MT}+$, these findings argue strongly against the idea that the change in retinal motion at eccentricities $>35^{\circ}$ can account for the modulation observed in the two pursuit-modulated regions in experiment 1. Indeed, when we applied the regression analysis of Equation 2 to all voxels in MT+, the data from experiment 2 showed a significant pursuit bias (i.e., $\beta_{4}>0$ ) for voxels in the medial and lateral pursuit regions (as in experiment 1), but none of these voxels nor any other voxels in $\mathrm{MT}+$ showed a significant correlation with pursuit amplitude (i.e., $\beta_{5}=0$ ), indicating that there were no significant influences of edge displacement. This result was obtained in all six subjects.

\section{Discussion}

Previous fMRI studies have identified two subdivisions of human $\mathrm{MT}+$ as putative homolog pairs of monkey MT and MST (Dukelow et al., 2001; Huk et al., 2002). pMT has retinotopic organization, with central vision located in ventral/posterior parts of MT. pMST lacks such retinotopic organization (Huk et al., 2002). Anterior to pMST, one finds an area responsive to pursuit in the absence of retinal flow (Dukelow et al., 2001). Pursuit signals and luminance-gradient signals interact within MT+ (Goltz et al., 2003), but no fMRI evidence exists for interaction between retinal motion and eye velocity signals. Our experiments tested these interactions, and we found functional differences in the role of pursuit signals across MT+.

Experiment 1 showed that the influence of pursuit is not homogeneous across MT+. First, the effect of pursuit on flowrelated responses was significantly larger for pMST than for pMT (Fig. 2). Second, we found two subregions in which the response to expanding retinal flow was strongly boosted by the pursuit task (Fig. 3). The medial one showed considerable overlap with pMST but did not encompass all of it (Fig. 4). This suggests that a substructure exists even within pMST. The lateral ROI fell mostly outside pMT and pMST. These new findings for human MT + are 
nicely in line with single-cell studies in monkeys, which show that some cells in MT and many neurons in MST modulate their responses to optic flow during pursuit (Bradley et al., 1996; Bremmer et al., 1997; Page and Duffy, 1999). Experiment 2 provides novel evidence that the interaction between retinal motion and pursuit in a subregion of PMST involves the computation of head-centric flow.

\section{Enhanced BOLD signal attributable to retinal or extra-retinal signals?}

In experiment 1 , we identified pursuit-modulated regions by selecting voxels with enhanced activation in the pursuit task compared with fixation. This means that voxels may have been selected for different reasons. (1) Voxels contain a mixture of pursuit- and flow-related units without interaction. During pursuit, both populations become active, resulting in enhanced BOLD signals compared with the fixation condition in which only flow-related units are active. (2) Voxels contain units that combine pursuit and flow signals; the interaction between pursuit and flow boosts the BOLD signal. (3) The retinal motions were not sufficiently matched during fixation and pursuit, causing more activation during pursuit.

We believe the last explanation is unlikely. In line with published behavior (Collewijn and Tamminga, 1984, 1986), our control experiments showed that pursuit was accurate (Fig. 10), yet minimal amounts of slip $(\sim 0.5 \%)$ cannot be avoided near reversals of target motion. Thus, the fovea representation in MT could be activated more during pursuit compared with fixation. Published MT retinotopy (Huk et al., 2002) suggests that the lateral pursuit region could perhaps overlap central MT (a ventral/posterior part of MT that possibly fell outside our pMT ROIs). If true, we would expect significant activation of this same region by visual flow compared with static dots. This was found in only two of our seven subjects (Fig. 5, lateral ROI). We believe, therefore, that the lateral pursuit region does not reflect a pure visual-flow-selective region corresponding to central MT. The medial pursuit region primarily overlaps pMST (Fig. 4). This region thus responds to high-speed flow in both the contralateral and ipsilateral visual periphery, i.e., a wide-field visual flow response, which makes it unlikely that small changes in visual flow attributable to pursuit errors (of $\sim 0.5^{\circ} / \mathrm{s}$ ) would modulate the BOLD response significantly in this area. Because a small portion of the flow field shifted in-and-out of view in the pursuit task, but not in the fixation task, one could still argue that enhanced activation during pursuit was caused by the change in retinal flow signals rather than by extraretinal pursuit signals. If true, the responses should depend strongly on the amplitude of the pursuit movements that determine the displacement of the stimulus window on the peripheral retina. Experiment 2 showed, however, that activity across MT + did not depend significantly on pursuit amplitude (Figs. 6, 9, 11), indicating that the change in retinal motion at eccentricities $>35^{\circ}$ cannot account for the observed activity modulation either.

To distinguish between independence and interaction of the responses to visual flow and pursuit, we investigated various combinations of retinal rotational flow and pursuit of the same frequency in experiment 2. By focusing on responses that depend on a $180^{\circ}$ phase difference between these signals, we could localize voxels with true interaction between flow and pursuit signals (see Results) (Figs. 6, 7). Neither pursuit errors nor edge effects could affect these results because low-frequency pursuit hardly shows phase errors (Fig. 10) (Collewijn and Tamminga, 1984,
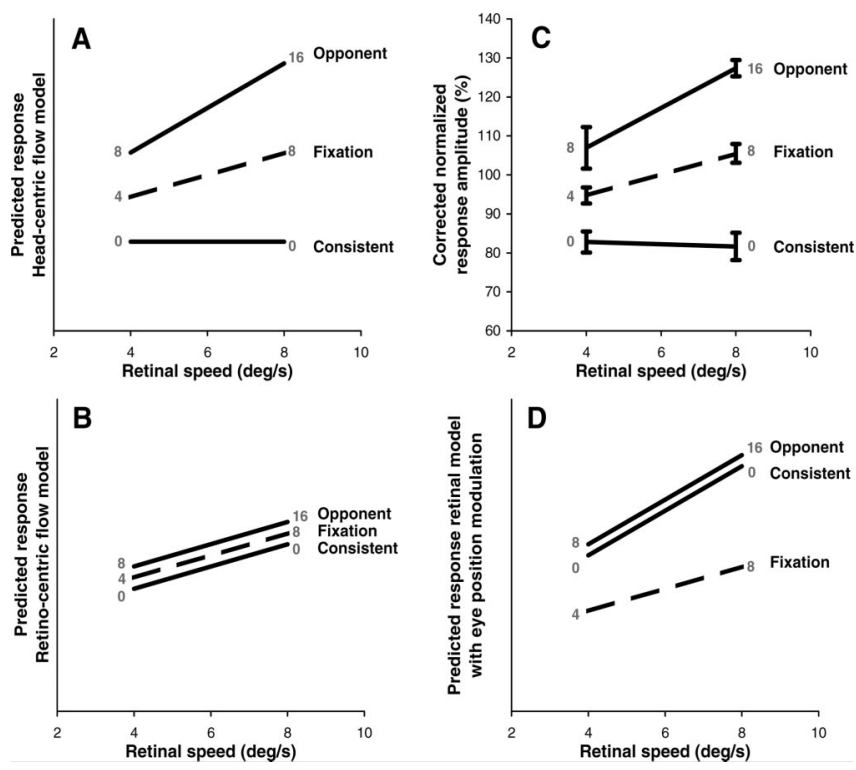

Figure 12. Comparison of data and models. $A$, Predicted response pattern for head-centric flow model. $\boldsymbol{B}$, Predicted parallel response lines for retino-centric flow model. $\boldsymbol{C}$, Amplitudes of the measured BOLD responses in experiment 2 , averaged across all subjects. Data from each subject were corrected for the pursuit bias and normalized with respect to the mean response amplitude. Error bars indicate \pm 1 SEM. $\boldsymbol{D}$, Interaction with eye position predicts identical responses for consistent and opponent pursuit that differ from the responses during fixation. Values in gray are head-centric rotation speeds (in degrees per second). Ordinate scaling in $A, B$, and $\boldsymbol{D}$ is in arbitrary units.

1986). Thus, in part of area pMST, the BOLD response is not caused by purely retinal signals but by an interaction between retinal signals and pursuit.

\section{Sensitivity to head-centric rotational flow}

The $180^{\circ}$ phase difference between pursuit and rotational flow in our opponent and consistent pursuit conditions caused a change in head-centric rotational speed of 8 or $16 \%$ while the retinocentric rotational flow speed and the eye pursuit speed was maintained (Fig. 1). In a region approximately corresponding to the medial pursuit area, BOLD activation showed a clear metric relation with the speed of the head-centric rotational flow (Fig. 8) and not with the speed of the retino-centric rotational flow (up to $8 \%$ ). Factorial analysis of the responses also showed sensitivity to pursuit (Fig. 9B) (pursuit bias $\beta_{4}>0$ ). To isolate the component of the BOLD signal related to head-centric speed, we subtracted the pursuit components [i.e., $\beta_{4} \times P b(t)$ and $\beta_{5} \times P s(t)$ in Eq. 2] from the BOLD signals. These corrected data were compared with the response patterns predicted by head-centric (Fig. 12A) and retino-centric (Fig. 12B) speed sensitivity. Note that there is a near perfect match between the data (Fig. 12C) and headcentric speed tuning. In contrast, interaction with eye position would predict identical BOLD signals for opponent and consistent conditions that differ from the fixation condition (Fig. 12D). This holds because, for either pursuit condition, a retinal rotational flow signal would combine with an eye position signal that is $\pm 90^{\circ}$ out of phase. Eye-position-dependent single-cell responses have been reported for macaque areas MT and MST (Bremmer et al., 1997), and DeSouza et al. (2002) reported modulation of BOLD signals by eye position in human MT + . However, DeSouza et al. (2002) found modulation of the baseline activity rather than of the response to expanding motion. Thus, 
there is no direct fMRI evidence for modulation by eye position of flow-related BOLD responses.

Our results strongly suggest that the activity in pHFR is quantitatively related to the combination of pursuit signals and visual flow into a representation of head-centric flow. Few studies report quantitative relations between BOLD signals and visual motion parameters. Rees et al. (2000) reported increasing BOLD responses in $\mathrm{MT}+$ as a function of motion coherence. Chawla et al. (1998) reported that BOLD responses to expanding motion in $\mathrm{MT}+$ vary as function of stimulus speed, but they made no attempts to dissociate between head-centric and retino-centric speed sensitivities. To our knowledge, we have, for the first time, established a quantitative relation between BOLD signals and a visual motion signal that refers to a different reference frame than the retina.

\section{Functional role of head-centric flow}

Head-centric flow signals how the head moves with respect to the world and how objects move relative to the head (Beintema and van den Berg, 1998). In general, flow patterns can be decomposed uniquely into rotational and translational components (Koenderink, 1986). Our visual stimulus contained both components. The computation of head-centric rotational speed could be useful to cross-calibrate visual and vestibular signals that measure the rotation of the head in space, or it could serve to supplement the semicircular canals signals, e.g., as they adapt for slow continuous rotation in one direction. The computation of head-centric translational flow is useful to perceive the heading direction (Warren and Hannon, 1988; Royden et al., 1992; van den Berg, 1992). Our fMRI study does not test a quantitative measure of this translational component. In general, the compensation for eye velocity can be done within a retino-centric receptive field (Bradley et al., 1996; Beintema and van den Berg, 1998; van den Berg et al., 2005). Monkey studies have investigated interactions between retinal motion and pursuit signals in the dorsal portion of the MST (Bradley et al., 1996; Lappe et al., 1996; Shenoy et al., 1999, 2002; Page and Duffy, 1999; Churchland and Lisberger, 2005) and the lateral portion of the MST (Thier and Erickson, 1992; Ilg et al., 2004; Churchland and Lisberger, 2005). Such interactions often concern gain modulation of the responses, and, less often, the interactions concern shifts of the tuning curve to compensate for the pursuit speed. These studies agree that an important role of the interaction between pursuit and visual flow may be the reconstruction of the visual motion relative to the head (or body).

We show that the interaction between pursuit and flow is not evenly distributed across human $\mathrm{MT}+$. Second, we find that modulation by pursuit (experiment 1) occurs in a subregion of pMST that overlaps with the region that shows head-centric flow sensitivity (experiment 2). It remains to be studied whether a similar subdivision with head-centric flow sensitivity can be found in monkey MT satellites MST and FST.

\section{References}

Bardy BG, Warren Jr WH (1997) Visual control of braking in goal-directed action and sport. J Sports Sci 15:607-620.

Barton JJ, Simpson T, Kiriakopoulos E, Stewart C, Crawley A, Cuthrie B, Wood M, Mikulis D (1996) Functional MRI of lateral occipitotemporal cortex during pursuit and motion perception. Ann Neurol 40:387-398.

Beintema JA, van den Berg AV (1998) Heading detection using motion templates and eye velocity gain fields. Vision Res 38:2155-2179.

Beintema JA, van den Berg AV (2001) Pursuit affects precision of perceived heading for small viewing apertures. Vision Res 41:2375-2391.
Bradley DC, Maxwell M, Andersen RA, Banks MS, Shenoy KV (1996) Mechanisms of heading perception in primate visual cortex. Science 273:1544-1547.

Bremmer F, Ilg UJ, Thiele A, Distler C, Hoffmann KP (1997) Eye position effects in monkey cortex. I. Visual and pursuit-related activity in extrastriate areas MT and MST. J Neurophysiol 77:944-961.

Britten KH, van Wezel RJ (1998) Electrical microstimulation of cortical area MST biases heading perception in monk. Nat Neurosci 1:59-63.

Chawla D, Phillips J, Buechel C, Edwards R, Friston KJ (1998) Speeddependent motion-sensitive responses in V5: an fMRI study. NeuroImage 7:86-96.

Churchland AK, Lisberger SG (2005) Relationship between extraretinal component of firing rate and eye speed in area MST of macaque monkeys. J Neurophysiol 94:2416-2426.

Collewijn H, Tamminga EP (1984) Human smooth and saccadic eye movements during voluntary pursuit of different target motions on different backgrounds. J Physiol (Lond) 351:217-250.

Collewijn H, Tamminga EP (1986) Human fixation and pursuit in normal and open-loop conditions: effects of central and peripheral retinal targets. J Physiol (Lond) 379:109-129.

Collewijn H, van der Mark F, Jansen TC (1975) Precise recording of human eye movements. Vision Res 15:447-450.

Crowell JA, Banks MS, Shenoy KV, Andersen RA (1998) Visual self-motion perception during head turns. Nat Neurosci 1:732-737.

de Jong BM, Shipp S, Skidmore B, Frackowiak RS, Zeki S (1994) The cerebral activity related to the visual perception of forward motion in depth. Brain 117:1039-1054.

Desimone R, Ungerleider LG (1986) Multiple visual areas in the caudal superior temporal sulcus of the macaque. J Comp Neurol 248:164-189.

DeSouza JF, Dukelow SP, Vilis T (2002) Eye position signals modulate early dorsal and ventral visual areas. Cereb Cortex 12:991-997.

Duffy CJ, Wurtz RH (1991) Sensitivity of MST neurons to optic flow stimuli. II. A continuum of response selectivity to large-field stimuli. J Neurophysiol 65:1329-1345.

Dukelow SP, DeSouza JF, Culham JC, van den Berg AV, Menon RS, Vilis T (2001) Distinguishing subregions of the human MT + complex using visual fields and pursuit eye movements. J Neurophysiol 86:1991-2000.

Dumoulin SO, Bittar RG, Kabani NJ, Baker Jr CL, Le Goualher G, Buce Pike G, Evans AC (2000) A new anatomical landmark for reliable identification of human area V5/MT: a quantitative analysis of sulcal patterning. Cereb Cortex 10:454-463.

Dupont P, Orban GA, De Bruyn B, Verbruggen A, Mortelmans L (1994) Many areas in the human brain respond to visual motion. J Neurophysiol 72:1420-1424.

Forman SD, Cohen JD, Fitzgerald M, Eddy WF, Mintun MA, Noll DC (1995) Improved assessment of significant activation in functional magnetic resonance imaging (fMRI): use of a cluster-size threshold. Magn Reson Med 33:636-647.

Gibson JJ (1966) The perception of the visual world. Boston, MA: Houghton Mifflin.

Goebel R, Khorram-Sefat D, Muckli L, Hacker H, Singer W (1998) The constructive nature of vision: direct evidence from functional magnetic resonance imaging studies of apparent motion and motion imagery. Eur J Neurosci 10:1563-1573.

Goltz HC, DeSouza JF, Menon RS, Tweed DB, Vilis T (2003) Interaction of retinal image and eye velocity in motion perception. Neuron 39:569-576.

Graziano MSA, Andersen RA, Snowden RJ (1994) Tuning of MST neurons to spiral motions. J Neurosci 14:54-67.

Hanada M (2005) An algorithmic model of heading perception. Biol Cybern 92:8-20.

Huk AC, Heeger DJ (2002) Pattern-motion responses in human visual cortex. Nat Neurosci 5:72-75.

Huk AC, Dougherty RF, Heeger DJ (2002) Retinotopy and functional subdivision of human areas MT and MST. J Neurosci 22:7195-7205.

Ilg UJ, Schumann S, Thier P (2004) Posterior parietal cortex neurons encode target motion in world-centered coordinates. Neuron 43:145-151.

Koenderink JJ (1986) Optic flow. Vision Res 26:161-179.

Koenderink JJ, van Doorn AJ (1987) Facts on optic flow. Biol Cybern $56: 247-254$. 
Lappe M (1998) A model of the combination of optic flow and extraretinal eye movement signals in primate extrastriate visual cortex. Neural Net 11:742-744.

Lappe M, Bremmer F, Pekel M, Thiele A, Hoffman K-P (1996) Optic flow processing in monkey STS: a theoretical and experimental approach. J Neurosci 16:6265-6285.

Lappe M, Bremmer F, van den Berg AV (1999) Perception of self-motion from visual flow. Trends Cogn Sci 3:329-336.

Maunsell JH, van Essen DC (1983) The connections of the middle temporal visual area (MT) and their relationship to a cortical hierarchy in the macaque monkey. J Neurosci 3:2563-2586.

McCarthy G, Spicer M, Adrignolo A, Luby M, Gore J, Allison T (1995) Brain activation associated with visual motion studied by functional magnetic resonance imaging in humans. Hum Brain Map 2:234-243.

Morrone MC, Tosetti M, Montanaro D, Fiorentini A, Cioni G, Burr DC (2000) A cortical area that responds specifically to optic flow, revealed by fMRI. Nat Neurosci 3:1322-1328.

Orban GA, Lagae L, Verri A, Raiguel S, Xiao D, Maes H, Torre V (1992) First-order analysis of optical flow in monkey brain. Proc Natl Acad Sci USA 89:2595-2599.

Orban GA, Fize D, Peuskens H, Denys K, Nelissen K, Sunaert S, Todd J, Vanduffel W (2003) Similarities and differences in motion processing between the human and macaque brain: evidence from fMRI. Neuropsychologia 41:1757-1768.

Page WK, Duffy CJ (1999) MST neuronal responses to heading direction during pursuit eye movements. J Neurophysiol 81:596-610.

Page WK, Duffy CJ (2003) Heading representation in MST: sensory interactions and population encoding. J Neurophysiol 89:1994-2013.

Perrone JA (1992) Model for the computation of self-motion in biological systems. J Opt Soc Am 9:177-194.

Petit L, Haxby JV (1999) Functional anatomy of pursuit eye movements in humans as revealed by fMRI. J Neurophysiol 82:463-471.

Peuskens H, Sunaert S, Dupont P, Van Hecke P, Orban GA (2001) Human brain regions involved in heading estimation. J Neurosci 21:2451-2461.

Rees G, Friston K, Koch C (2000) A direct quantitative relationship between the functional properties of human and macaque V5. Nat Neurosci 3:716-723.

Regan D, Beverley KI (1982) How do we avoid confounding the direction we are looking with the direction we are moving? Science 215:194-196.

Royden CS, Banks MS, Crowell JA (1992) The perception of heading during eye movements. Nature 360:583-585.

Royden CS, Crowell JA, Banks MS (1994) Estimating heading during eye movements. Vision Res 34:3197-3214.

Shenoy KV, Bradley DC, Andersen RA (1999) Influence of gaze rotation on the visual response of primate MSTd neurons. J Neurophysiol 81:2764-2786.

Shenoy KV, Crowell JA, Andersen RA (2002) Pursuit speed compensation in cortical area MSTd. J Neurophysiol 88:2630-2647.

Smith AT, Greenlee MW, Singh KD, Kraemer FM, Henning J (1998) The processing of first- and second-order motion in human visual cortex assessed by functional magnetic resonance imaging (fMRI). J Neurosci 18:3816-3830.

Strupp JP (1996) Stimulate: a GUI-based fMRI analysis software package. NeuroImage 3:S607.

Sunaert S, Van Hecke P, Marchal G, Orban GA (1999) Motion-responsive regions of the human brain. Exp Brain Res 127:355-370.

Talairach J, Tournoux P (1988) Co-Planar stereotaxic atlas of the human brain: 3-dimensional proportional system: an approach to cerebral imaging. Stuttgart, Germany: Thieme Medical Publishers.

Tanabe J, Tregellas J, Miller D, Ross RG, Freedman R (2002) Brain activation during smooth-pursuit eye movements. NeuroImage 17:1315-1324.

Thier P, Erickson RG (1992) Responses of visual-tracking neurons from cortical area MST-I to visual, eye and head motion. Eur J Neurosci 4:539-553.

Tootell RB, Reppas JB, Dale AM, Look RB, Sereno MI, Malach R, Brady JT, Rosen BR (1995a) Visual motion aftereffect in human cortical area MT revealed by functional magnetic resonance imaging. Nature 375:139-141.

Tootell RB, Reppas JB, Kwong KK, Malach R, Born RT, Brady TJ, Rosen BR, Belliveau JW (1995b) Functional analysis of human MT and related visual cortical areas using magnetic resonance imaging. J Neurosci 15:3215-3230.

Upadhyay UD, Page WK, Duffy CJ (2000) MST responses to pursuit across optic flow with motion parallax. J Neurophysiol 84:818-826.

van den Berg AV (1992) Robustness of perception of heading from optic flow. Vision Res 32:1285-1296.

van den Berg AV (1996) Judgements of heading. Vision Res 36:2337-2350.

van den Berg AV, Beintema JA (2000) The mechanism of interaction between visual flow and eye velocity signals for heading perception. Neuron 26:747-752.

van den Berg AV, Beintema JA, Frens MA (2001) Heading and path percepts from visual flow and eye pursuit signals. Vision Res 41:3467-3486.

van den Berg AV, van Ee R, Noest AJ (2005) Mixed visual reference frames: perceiving nonretino-centric visual quantities in a retino-centric frame. Proceedings of the International Society for Optical Engineering, Human Vision and Electronic Imaging X, Vol 5666 (Rogowitz BE, Pappas TN, Daly SJ, eds), pp 449-461. Bellingham, WA: SPIE.

Warren Jr WH (1995) Self-motion: visual perception and visual control. In: Perception of space and motion. San Diego: Academic.

Warren Jr WH, Hannon DJ (1988) Direction of self-motion is perceived from optical flow. Nature 336:162-163.

Watson JD, Myers R, Frackowiak RS, Hajnal JV, Woods RP, Mazziotta JC, Shipp S, Zeki S (1993) Area V5 of the human brain: evidence from a combined study using positron emission tomography and magnetic resonance imaging. Cereb Cortex 3:79-94.

Zeki S, Watson JD, Lueck CJ, Friston KJ, Kennard C, Frackowiak RS (1991) A direct demonstration of functional specialization in human visual cortex. J Neurosci 11:641-649.

Zemel RS, Sejnowski TJ (1998) A model for encoding multiple object motions and self-motion in area MST of primate visual cortex. J Neurosci 18:531-547. 